\title{
Two rare cases of node-positive differentiated gastric cancer despite their infiltration to sm1, their small size, and lack of lymphatic invasion into the submucosal layer
}

\author{
Hideki Nagano ${ }^{1,3}$, Shigekazu Ohyama ${ }^{1}$, Tetsu Fukunaga ${ }^{1}$, Naoki Hiki $^{1}$, Yasuyuki Seto $^{1}$, \\ ToshiharU YAMAGUChI ${ }^{1}$, Yo $\mathrm{KaTO}^{2}$, and AKIO YAMAGUChI ${ }^{3}$ \\ ${ }^{1}$ Department of Surgery, Cancer Institute Hospital of JFCR, Tokyo, Japan \\ ${ }^{2}$ Department of Pathology, Cancer Institute Hospital of JFCR, Tokyo, Japan \\ ${ }^{3}$ First Department of Surgery, Faculty of Medicine, University of Fukui, 23-3 Shimoaizuki, Matsuoka, Eiheiji-cho, Yoshida, Fukui 910-1193, \\ Japan
}

\begin{abstract}
Early gastric cancer without lymph node metastasis has been reported after the analysis of many cases, and a consensus has been reached about this condition. We report two cases of nodepositive differentiated $\operatorname{sm} 1$ gastric cancer without lymphatic invasion into the submucosal layer. Case 1 was a 73-year-old man who underwent EMR for 0-IIc early gastric cancer (EGC) on the gastric angle, with a histological diagnosis of tub1. Pathological examination revealed a 0-IIc lesion that was $12 \mathrm{~mm}$ in size and $\mathrm{sm1}$ in invasion depth without lymphaticvascular invasion. However, the infiltration in the submucosal layer was relatively wide. The patient subsequently underwent distal gastrectomy with D2 lymph node dissection. Pathological examination revealed level 2 lymph node metastasis. Case 2 was a 62-year-old woman who underwent ER for a 0 -I+IIctype EGC on the greater curvature of the antrum, with a histological diagnosis of tub1. Pathological examination revealed a 0 -I+IIc-type lesion that was $15 \mathrm{~mm}$ in size and sm1 in depth. Lymphatic invasions in the muscularis mucosa were found, but none were seen in the submucosal layer. Two years later, follow-up computed tomography (CT) showed a lymph node swelling in the infrapyloric region. Distal gastrectomy with D2 dissection was then performed, and pathological examination revealed level 1 lymph node metastasis. Although the lesions in both patients satisfied the criteria of Gotoda et al. for minimal risk of nodal involvement, lymph node metastasis was observed in these patients. Curative surgery with lymph node dissection is thus required in patients with wide infiltration of the submucosal layer or lymphatic invasion in the muscularis mucosa.
\end{abstract}

Key words Gastric cancer · sm1 Infiltration depth · Lymph node metastasis

Offprint requests to: $\mathrm{H}$. Nagano

Received: March 18, 2007 / Accepted: November 13, 2007

\section{Introduction}

Because lymph node metastasis is an important prognostic factor for patients with early gastric cancer (EGC), treatment strategy is determined according to the risk of nodal involvement. For patients with mucosal gastric cancer, much evidence has been reported supporting the idea that a limited subgroup of these patients is free from the risk of lymph node metastasis [1]. However, for patients with infiltration into the surface of the submucosal layer, the risk of metastasis has been uncertain. Recently, the risk of lymph node metastasis from EGC was analyzed in a large collaborative study sponsored by the National Cancer Center and our institute [2]. According to their data, none of $145 \mathrm{sm} 1$ (cancer with submucosal invasion no more than $500 \mu \mathrm{m}$ in depth) lesions were associated with lymphatic metastasis under the following conditions: lesions no more than $30 \mathrm{~mm}$ in size, differentiated histology, and lack of lymphatic or venous involvement. For the patients whose disease satisfied these conditions, it was considered permissible to apply endoscopic mucosal resection (EMR) and to omit lymph node dissection. In the two patients we report here, sm1 lesions were accompanied by lymph node metastasis, despite the lesions satisfying the strict conditions noted above. We report herein two rare cases of EGC that we encountered after completion of this collaborative study. All technical terms used in this report are in accordance with the Japanese classification of gastric carcinoma (second English edition) [3].

\section{Report of cases}

\section{Case 1}

Case 1 was a 73-year-old man who had received upper gastrointestinal endoscopy for stomach cancer screen- 
ing at our center every 4 years, and no abnormalities had been pointed out until 2001. In August 2002, when he had still no gastric complaints, a depressed lesion (0-IIc) on the lesser curvature of the gastric angle was revealed by the examination (Fig. 1). A biopsy showed a well-differentiated tubular adenocarcinoma (tub1). The patient underwent EMR in September 2002. In this procedure, physiological saline was injected into the submucosal layer to elevate the lesion, which was treated by piecemeal resection and completely removed macro-

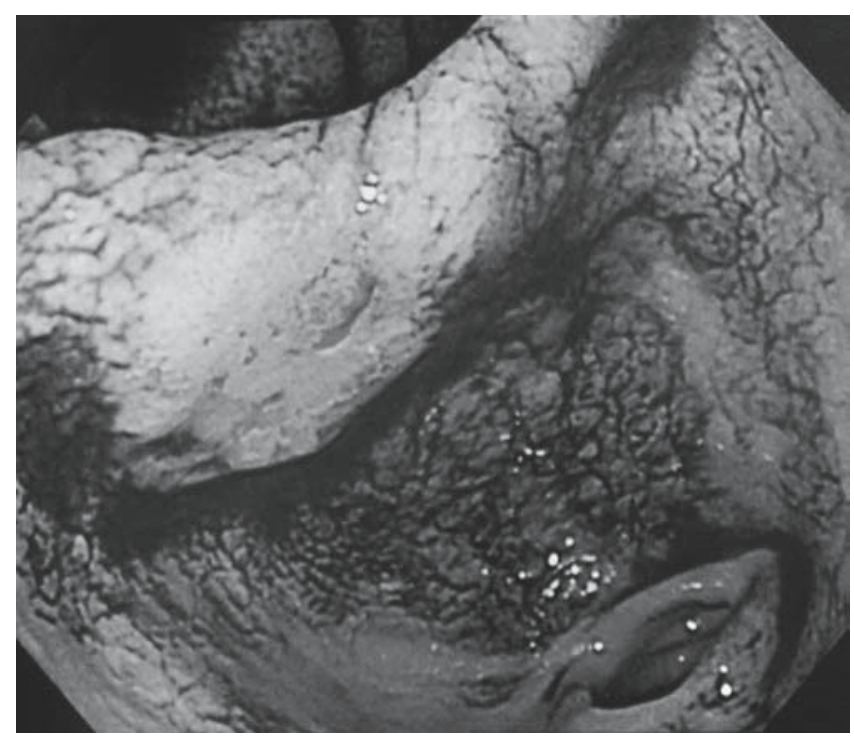

Fig. 1. Upper gastrointestinal endoscopy revealed a depressed lesion (0-IIc) on the lesser curvature of the gastric angle. A histological diagnosis of well-differentiated tubular adenocarcinoma (tub1) was made upon biopsy scopically. The gross appearance of the tumor was 0-IIctype EGC. Microscopically, it was a well-differentiated tubular adenocarcinoma (tub1) with sm1 infiltration, $12 \mathrm{~mm}$ in size, ly0, v0, INF $\beta$, VM(-), EB, but the lateral margin was uncertain because of the difficulty of reconstructing the pieces following the piecemeal resection. No components of poorly differentiated adenocarcinoma were found. Abdominal computed tomography (CT) did not reveal a swollen lymph node or liver metastasis. The tumor infiltration was limited to slight invasion of the submucosal layer. The depth of submucosal invasion was about $200 \mu \mathrm{m}$, but the invasion was about $1000 \mu \mathrm{m}$ in width. Lymphatic-vessel involvement was not found (Fig. 2). Because the infiltration was spread over a relatively wide range and the lateral cut margin was unclear, pathologists recommended that the patient receive an additional surgical treatment. Accordingly, in November 2002, he underwent distal gastrectomy with D2 lymph node dissection. Histological examination of the resected specimen revealed no remnant cancer, but two positive lymph nodes were noted along the lesser curvature (no.3) and along the proximal part of the splenic artery (no.11p; 2/16), and this was diagnosed as level 2 lymph node metastasis. After an uneventful recovery, the patient was discharged from the hospital, and no cancer recurrence has been found 4.8 years after the gastrectomy.

\section{Case 2}

A 62-year-old woman had received upper gastrointestinal endoscopy for stomach cancer screening since 1997. In June 1999, a protruding lesion with an irregular

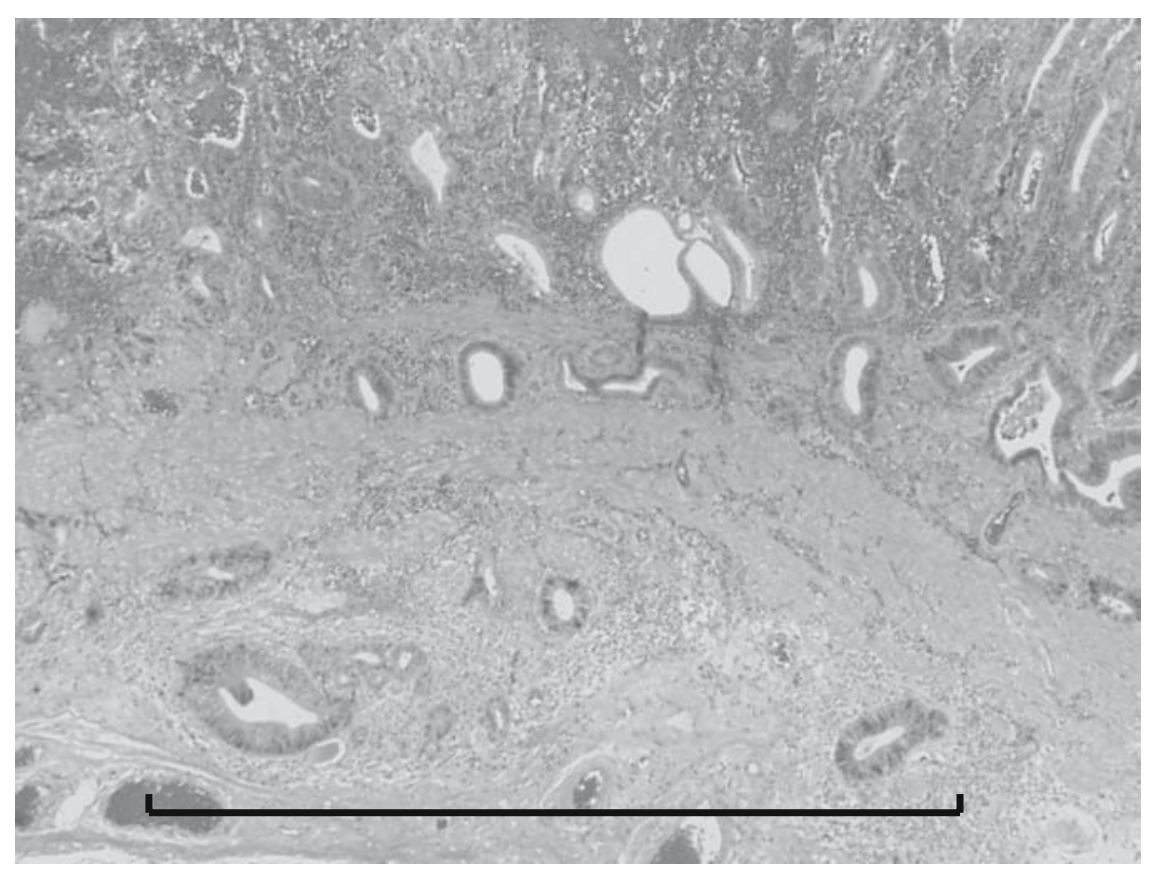

Fig. 2. Pathological examination revealed that the early gastric cancer consisted of tubular adenocarcinoma (tub1) with penetration to the superficial submucosal layer. No components of poorly differentiated adenocarcinoma were found. The depth of submucosal invasion was about $200 \mu \mathrm{m}$, but the invasion was about $1000 \mu \mathrm{m}$ in width. Lymphatic-vessel involvement was not found. $\mathrm{H} \& \mathrm{E}$, scale bar $1000 \mu \mathrm{m}$ 
central depression, associated with conversion of the mucosal fold on the greater curvature of the antrum, was pointed out by the examination (Fig. 3). A biopsy revealed a well-differentiated tubular adenocarcinoma (tub1). She underwent ER in June 1999. The tumor was removed in one piece, and the gross appearance was 0-I+IIc-type EGC. The tumor consisted of a well-

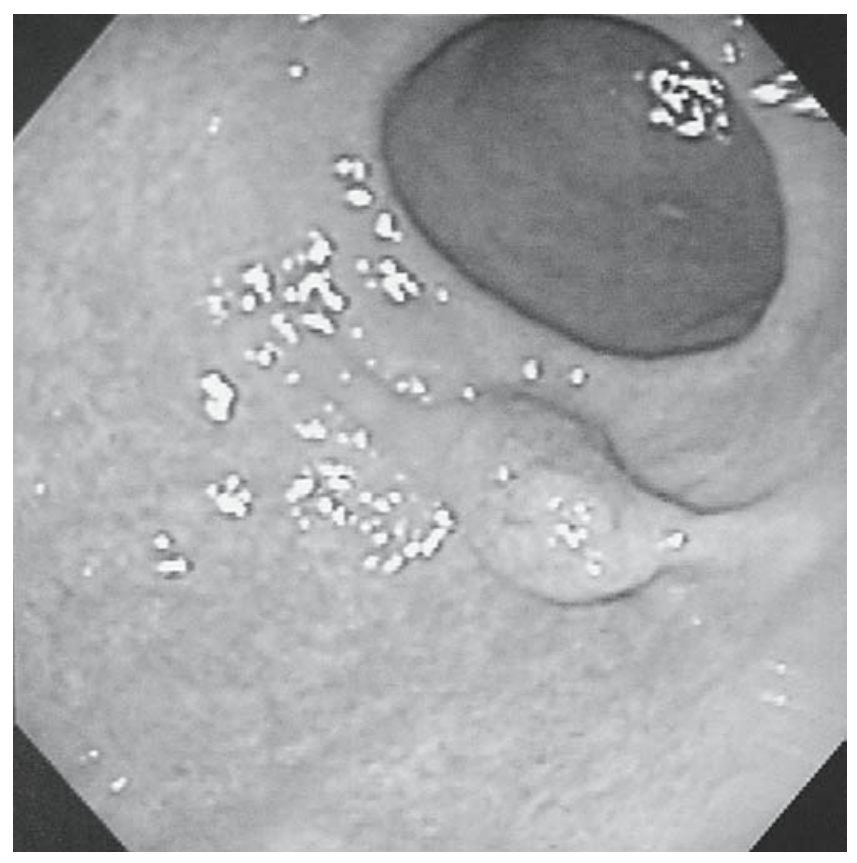

Fig. 3. Upper gastrointestinal endoscopy revealed a protruding tumor with an irregular central depression on the greater curvature of the antrum. A histological diagnosis of welldifferentiated tubular adenocarcinoma (tub1) was made upon biopsy. Endoscopic resection was done by one-piece resection differentiated tubular adenocarcinoma (tub1), $15 \mathrm{~mm}$ in size, ly0, v0, INF $\beta, \mathrm{VM}(-), \mathrm{LM}(-), \mathrm{EB}$, showing sm1 infiltration No components of poorly differentiated adenocarcinoma were found. The tumor, which infiltrated the surface of the submucosal layer (about $100 \mu \mathrm{m})$, had also a few lymphatic invasions in the muscularis mucosa, but no lymphatic invasion was found in the submucosal layer (Fig. 4).

The patient was followed up carefully, and 2 years later an abdominal CT revealed lymph node swelling in the infrapyloric region (no. 6), which was diagnosed as lymph node metastasis. We then recommended a surgical operation, and she underwent distal gastrectomy with D2 lymph node dissection in July 2001. Histological examination of the resected specimen revealed no remnant cancer around the ER-treated scar, but three positive lymph nodes were found in the infrapyloric region (no.6; 3/26). A diagnosis of level 1 lymph node metastasis was confirmed. After an uneventful recovery, she was discharged from the hospital and has had no recurrence 5 years after the gastrectomy.

\section{Discussion}

Endoscopic resection (ER) has been accepted as curative resection for early gastric cancer (EGC) without lymph node metastasis for a long time since its introduction. It is well known that lesions satisfying the following criteria are free of the risk of lymph node metastasis: intramucosal differentiated tumor; elevated lesion less than $30 \mathrm{~mm}$ in size, or depressed lesion less than $10 \mathrm{~mm}$; and lesions without an ulcer or ulcer scar. Recently,

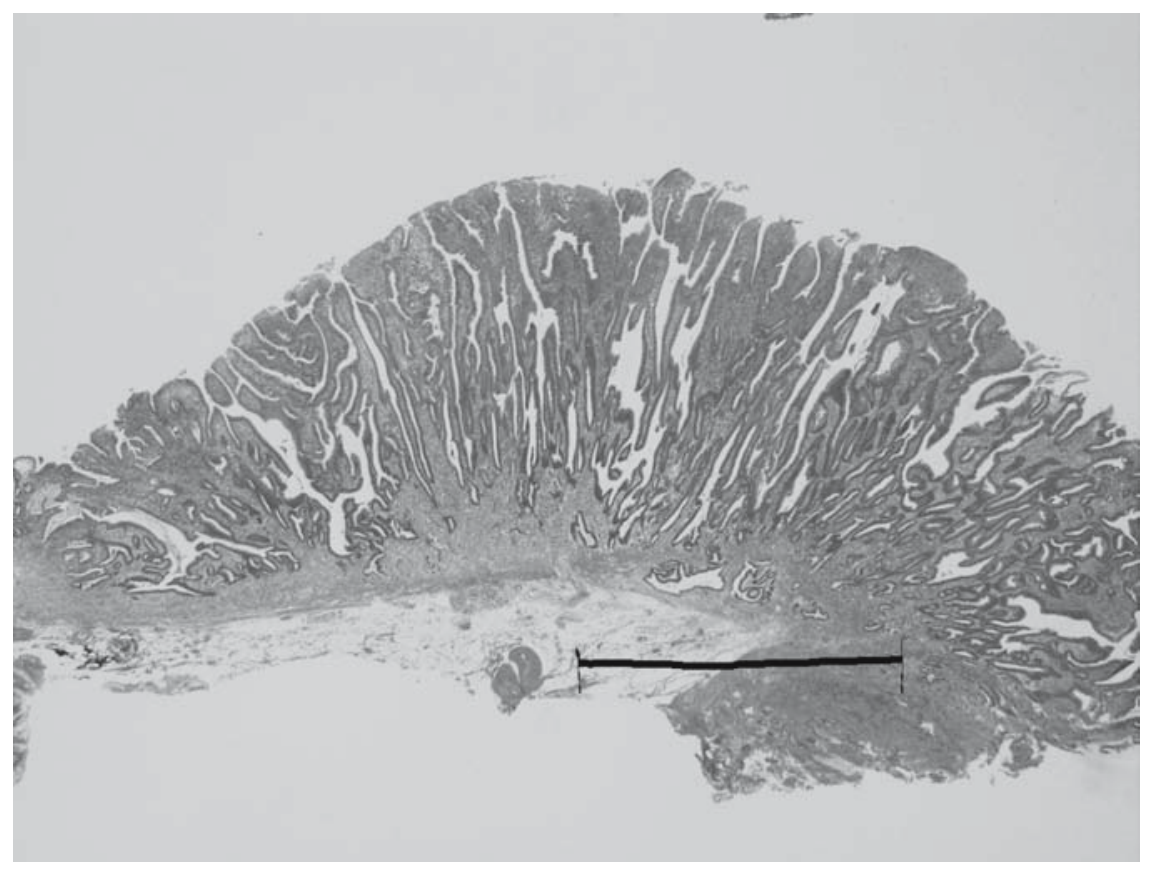

Fig. 4. Pathological examination revealed that the early gastric cancer consisted of tubular adenocarcinoma (tub1) with minute penetration to the superficial submucosal layer. The depth of submucosal infiltration was within $100 \mu \mathrm{m}$, and a few lymphatic invasions were revealed in the muscularis mucosa. No lymphatic invasion was found in the submucosal layer. No components of poorly differentiated adenocarcinoma were found. $\mathrm{H} \& \mathrm{E}$, scale bar $3000 \mu \mathrm{m}$ 
the development of endoscopic submucosal dissection (ESD) using an insulated-tip knife has made it possible for us to resect larger lesions, even those more than $30 \mathrm{~mm}$ in diameter, in differentiated tumors confined to the mucosal layer. As the indications for ESD are being widened, treatment indications for EGC with minute submucosal invasion have become the subject of debate. The degree of submucosal invasion is recognized as the most significant risk factor correlating with nodal involvement, while lymphatic invasion and histologic differentiation are also important risk factors for nodal involvement $[4,5]$. On the other hand, several reports have demonstrated the safety from lymphatic metastasis in a limited subgroup of sm1 gastric cancers [6-11]. This has raised the question of whether distal or total gastrectomy with lymph node dissection [12-14] is necessary for small, differentiated gastric cancers with a depth of sm1.

Recently Gotoda et al. [2] reported the incidence of lymph node metastasis after investigating 5265 EGCs at the National Cancer Center in Japan and our institute. They demonstrated the safety of endoscopic treatment for cancers with infiltration to the superficial submucosal layer. Among 1230 differentiated intramucosal tumors, lesions measuring less than $30 \mathrm{~mm}$ in size were not associated with lymph node metastasis, despite the presence or absence of scar ulcer, and those without ulceration did not show nodal involvement despite their size. In addition, none of 145 differentiated cancers less than $30 \mathrm{~mm}$ in diameter without lymphatic-vascular involvement were associated with lymph node metastasis to the extent that the penetration was limited to within $500 \mu \mathrm{m}$ into the submucosal layer (sm1). In Japan, this study of Gotoda et al. [2] has been used frequently in decision-making for additional surgical treatment following pathological examinations after ESD, and the data have been used as baseline in the development of gastric cancer treatment guidelines as well.

The width of infiltration in the submucosal layer and lymphatic involvement in the mucosal layer have not been described in detail in earlier reports, but Ishigami et al. [15] demonstrated a significant correlation between lymphatic invasion and the degree of submucosal invasion, not only regarding the depth of invasion but also with the width of tumor infiltration. Listrom et al. [16] have provided a detailed description of the anatomical distribution of gastric lymphatics, and have shown that lymphatic vessels are also found in the lower third of the lamina propria or muscularis mucosa. Concerning lymphatic-vascular involvement, pathological reports commonly mention the status below the mucosal layer. In the report of Gotoda et al. [2], which was the collaborative study sponsored by the National Cancer Center and our institute, lymphatic-vascular permeation was also evaluated within the submucosal layer, but not in the lamina propria, and this permeation within the submucosal layer had been confirmed by a pathologist at our institute.

In the present study, both patients were treated with EMR, as according to the criteria of Gotoda et al. [2], their lesions were associated with a minimal risk of lymph node metastasis. In case 1 , evaluation of the lateral margin was difficult because of the piecemeal resection. Precise pathological evaluation, carried out at 2-mm intervals, showed an sm1 lesion $12 \mathrm{~mm}$ in diameter, without vessel permeation in the submucosal layer; however, it cannot be denied that we might have overlooked the presence of lymphatic invasion in the layer because of a "burning" effect on the resection margin following the piecemeal resection. In case 2, the lesion, treated with en-bloc resection, was $15 \mathrm{~mm}$ in size, in which lymphatic invasion was observed in the lamina propria, but not in the submucosal layer. It is considered that evaluation of the differentiation type at the deepest invasive site of a carcinoma is essential. In the present study, neither patient had any poorly differentiated adenocarcinoma components; the lesions consisted only of well-differentiated components.

In 2000, following the publication of the report of Gotoda et al. [2], we carried out a study in which we examined details of $70 \mathrm{sm} 1$ well-differentiated gastric cancers in 2355 patients who had undergone EMR at our hospital. Of these $70 \mathrm{sm} 1$ cases, lymphatic-vascular invasion was observed in 13 cases, and it was not found in 57 cases. Lymph node metastasis was present in 3 of the 57 cases without lymphatic-vascular invasion (5.3\%); 2 of these cases have been presented here-in the other case, the lesion was $63 \mathrm{~mm}$ in size. Nodal involvement was present in 4 of the 13 cases with lymphatic-vascular invasion (31\%). Among sm1 cancers no more than $30 \mathrm{~mm}$ in size showing differentiated histology, only the 2 cases presented here showed lymph node metastasis. Therefore, the 2 cases presented here were considered to be extremely rare.

Given that these cases are rare but existing, we should not determine the risk of lymph node metastasis in cases of sm1 gastric cancer based only upon the lesion size or extent of lymphatic involvement in the submucosal layer or upon the degree of tumor differentiation. More careful evaluation should be carried out, investigating additional characteristics such as the width of submucosal invasion or the extent of lymphatic involvement in the mucosal layer.

From these clinical observations, it is too early to endorse the complete applicability of ER in all cases of sm1 gastric cancer. Careful follow-up with the use of annual abdominal CT scans is required for all types of sm1 gastric cancer for the early detection of lymph node metastasis. When lymph node metastasis is suspected, additional surgical treatment should be required. 


\section{References}

1. Japanese Gastric Cancer Association. The guideline for gastric cancer therapy (in Japanese). Tokyo: Kanehara, 2001.

2. Gotoda T, Yanagisawa A, Sasako M, Ono H, Nakanishi Y, Shimoda T, et al. Incidence of lymph node metastasis from early gastric cancer: estimation with a large number of cases at two large centers. Gastric Cancer 2000;3:219-25.

3. Japanese Gastric Cancer Association. Japanese classification of gastric carcinoma, second English edition. Gastric Cancer 1998;1: $20-4$.

4. Kurihara N, Kubota T, Otani Y, Ohgami M, Kumai K, Sugiura $\mathrm{H}$, et al. Lymph node metastasis of early gastric cancer with submucosal invasion. Br J Surg 1998;85:835-9.

5. Shimoyama S, Yasuda H, Mafune K, Kaminishi M. Indications of minimized scope of lymphadenectomy for submucosal gastric cancer. Ann Surg Oncol 2002;9:625-31.

6. Aoyagi K, Kohfuji K, Yano S, Murakami N, Hori H, Terasaki Y, et al. Submucosal gastric cancer with lymph node metastasis a clinicopathological study. Kurume Med J 1999;46:171-4.

7. Yasuda K, Shiraishi N, Suematsu T, Yamaguchi K, Adachi Y, Kitano S. Rate of detection of lymph node metastasis is correlated with the depth of submucosal invasion in early stage gastric carcinoma. Cancer 1999;85:2119-23.

8. Mishima T, Chonan A, Ando M, Tamura T, Kusaka T, Noguchi $\mathrm{T}$, et al. The possibility of expanding the indications for endoscopic mucosal resection of early gastric cancer (in Japanese with English abstract). Gastroenterol Endosc 2001;43:1257-67.
9. Matuszaki H, Kikuchi S, Kakita A. Evaluation of the morphology of submucosal tumor invasion and its volume in early gastric cancer. In vivo 2003;17:41-4.

10. Amano Y, Ishihara S, Amano K, Hirakawa K, Adachi K, Fukuda $\mathrm{R}$, et al. An assessment of local curability of endoscopic surgery in early gastric cancer without satisfaction of current therapeutic indications. Endoscopy 1998;30:548-52.

11. Yamada H, Nihei Z, Yamashita T, Shirota Y, Ichikawa W, Sugihara K. Is lymphadenectomy needed for all submucosal gastric cancers? Eur J Surg 2001;167:199-203.

12. Yoshikawa T, Tsuburaya A, Kobayashi O, Sairenji M, Motohashi $\mathrm{H}$, Noguchi Y. Is D2 lymph node dissection necessary for early gastric cancer? Ann Surg Oncol 2002;9:401-5.

13. Okamura T, Tsujitani S, Korenaga D, Haraguchi M, Baba H, Hiramoto Y, et al. Lymphadenectomy for cure in patients with early gastric cancer and lymph node metastasis. Am J Surg 1988; $155: 476-80$

14. Maruyama K, Okabayashi K, Kinoshita T. Progress in gastric cancer surgery in Japan and its limit of radicality. World J Surg 1987:11:418-25.

15. Ishigami $\mathrm{S}$, Natsugoe $\mathrm{S}$, Hokita $\mathrm{S}$, Tokushige $\mathrm{M}$, Saihara $\mathrm{T}$, Watanabe $\mathrm{T}$, et al. Carcinomatous lymphatic invasion in early gastric cancer invading into the submucosa. Ann Surg Oncol 1999;6:286-9.

16. Listrom MB, Fenoglio-Preiser CM. Lymphatic distribution of the stomach in normal, inflammatory, hyperplastic, and neoplastic tissue. Gastroenterology 1987;93:506-14.

\section{Editorial comment}

\section{A word of caution regarding easy expansion of the indications for endoscopic submucosal dissection in early gastric cancer}

Mitsuru Sasako ${ }^{1}$ and Tadakazu Shimoda ${ }^{2}$

${ }^{\mathrm{I}}$ Department of Surgery, Hyogo College of Medicine, 1-1

Mukogawa-cho, Nishinomiya, Hyogo 663-8501, Japan

${ }^{2}$ Division of Pathology, Central Laboratory Department, National

Cancer Center Hospital, Tokyo, Japan

With recent improvements in resection technique using the insulated-tip (IT) knife, hook knife, and flex knife, the number of endoscopic submucosal dissections (ESDs) and the proportion of one-piece resections have dramatically increased and the local recurrence rate has decreased. Consequently, there are moves to expand the indications for ESD, according to the article by T. Gotoda et al. [1]. However, both pathologists and clinicians should be prudent in deciding that additional surgery is not necessary.

In this issue of the journal, H. Nagano et al. [2] reported two rare cases in which unexpected lymph node metastases were found by additional surgery after ESD. In the past, as mentioned in this article, pathologists did not usually check for lymphatic invasion in the mucosal layer or in the muscularis mucosae, but in fact, a few patients showing such involvement had lymph node metastases. At the National Cancer Center Hospital, Tokyo, we had a patient with lymphatic invasion in the muscularis mucosae which, by deeper-cut examination, was found to connect to submucosal lymphatic invasion. Lymph node metastasis was discovered in this patient. In another patient with 20-mm IIa without lymphatic invasion, submucosal invasion within $500 \mu \mathrm{m}$ ( $\mathrm{sm} 1$ invasion) was discovered, and he too had lymph node metastasis. Histologically, the invaded portion in the submucosal layer was of the poorly differentiated type in a small area. Based on the report by Gotoda et al., such a patient could be followed up without additional surgery. However, these patients actually had nodal metastasis. Some authors have reported that lesions with wide submucosal invasion showed a greater tendency to have nodal metastasis. The correlation between the width and depth of submucosal invasion and their relationship with nodal metastasis should be further studied, as in colon cancer.

With regard to endoscopically resected material, pathologists should carefully examine the mucosal layer and the muscularis mucosae to exclude lymphatic invasion. If there is submucosal invasion, even minute, the histological grade should be precisely determined, and if it includes even a small portion of poorly differentiated histology, additional surgery should be considered. 
Otherwise, detailed informed consent, with explanation of the risk of nodal metastasis, and careful observation including $\mathrm{CT}$ scan are mandatory.

\section{References}

1. Gotoda T, Yanagisawa A, Sasako M, Ono H, Nakanishi Y, Shimoda $\mathrm{T}$, et al. Incidence of lymph node metastasis from early gastric cancer: estimation with a large number of cases at two large centers. Gastric Cancer 2000;3:219-25.

2. Nagano H, Ohyama S, Fukunaga T, Hiki N, Seto Y, Yamaguchi $\mathrm{T}$, et al. Two rare cases of node-positive differentiated gastric cancer despite their infiltration to sm1, their small size, and lack of lymphatic invasion into the submucosal layer. Gastric Cancer 2008; 11:53-8. 